\title{
CORRIGENDUM
}

'On the a.c. electrical conductivity of a Lorentz gas',

by J.H.M. Fu and R. S. B. ONG, J. Plasma Phys. 2, 1968, p. 1.

\section{Calculation of the electrical conductivity}

The current density is expressed by

$$
\mathbf{j}(\mathbf{k}, \omega)=-e \int_{-\infty}^{\infty} d^{3} \mathbf{v} \mathbf{v}\left[f_{0}(v)+g(\mathbf{k}, \mathbf{v}, \omega)\right] .
$$

Using the dimensionless velocity $\mathbf{c}=(m / 2 \theta)^{\frac{1}{2}} \mathbf{v}$ and noting that the current density associated with the equilibrium distribution function $f_{0}$ vanishes, we can write

$$
\mathbf{j}(\mathbf{k}, \omega)=-e\left(\frac{2 \theta}{m}\right)^{2} \int_{-\infty}^{\infty} d^{3} \operatorname{cc} g(\mathbf{k}, \mathbf{c}, \omega),
$$

$g(\mathbf{k}, \mathbf{c}, \omega)$ is given formally by

$$
g(\mathbf{k}, \mathbf{c}, \omega)=g_{n s}(\mathbf{k}, \mathbf{c}, \omega)+g_{s}(\mathbf{k}, \mathbf{c}, \omega) \delta(\mathbf{l}-\lambda \hat{\mathbf{k}} . \mathbf{c}),
$$

where $g_{n s}(\mathbf{k}, \mathbf{c}, \omega)$ is given by (2.15) in the original paper, and

$$
g_{s}(\mathbf{k}, \mathbf{c}, \omega)=\frac{e n_{0} E}{2 \pi^{\frac{3}{2}} A m}\left(\frac{m}{2 \theta}\right)^{\frac{1}{2}} c^{2} \mathbf{k} \cdot \mathbf{c} f_{0}^{\prime}(c) \delta(1-\lambda \hat{\mathbf{k}} \cdot \mathbf{c}),
$$

with $\lambda=(2 \theta / m)^{\frac{1}{2}} /(\omega / k)$ and $\delta$ the Dirac delta function.

Thus the complete expression for the current is given by the sum of two integrals. The first integral is given by (3.3) in the original paper, while the second integral is obtained by substituting (3) into (1) above. For a Maxwellian equilibrium distribution we get for the second integral

$$
\begin{aligned}
\mathbf{j}_{s}=-e\left(\frac{2 \theta}{m}\right)^{2} \int_{-\infty}^{\infty} & d^{3} \mathbf{c c} g_{s}(\mathbf{k}, \mathbf{c}, \omega) \\
= & \frac{e^{2} n_{0}}{\sqrt{(\pi) A m}}\left(\frac{2 \theta}{m}\right)^{\frac{3}{2}} \frac{1}{\lambda^{3}} E \mathbf{k}\left[\left(\frac{1}{\lambda^{3}}+\frac{3}{2 \lambda}\right) e^{-1 / \lambda^{2}}+\frac{3 \sqrt{ } \pi}{4} \operatorname{erfc}\left(\frac{1}{\lambda}\right)\right] .
\end{aligned}
$$

Comparing the resulting expression for the current with Ohm's law we obtain the a.c. electrical conductivity tensor. For a Maxwellian equilibrium distribution this is given by equations (3.5), (3.8) and (3.9) in the original paper plus the following additional expression resulting from (4) above:

$$
\boldsymbol{\sigma}_{s}=\frac{e^{2} n_{0}}{\sqrt{(\pi) A m}}\left(\frac{2 \theta}{m}\right)^{\frac{8}{2}} \frac{1}{\lambda^{3}} \mathbf{I}\left[\left(\frac{1}{\lambda^{3}}+\frac{3}{2 \lambda}\right) e^{-1 / \lambda^{2}}+\frac{3 \sqrt{ } \pi}{4} \operatorname{erfc}\left(\frac{1}{\lambda}\right)\right] .
$$

It is seen from (5) that for $\lambda \ll 1$ the contribution of $\sigma_{s}$ to the total electrical conductivity is negligibly small. For the extremely special case when $\omega$ and $k$ are both equal to zero we have the spatially homogeneous d.c. case and our basic equation reduces to that of Spitzer. The electrical conductivity is then given by (3.12) in the original paper. 